\title{
Neurocritical Care Has Matured and it is Time to Raise the Bar...Yet Again
}

\author{
Jose I. Suarez
}

๑ 2018 Springer Science+Business Media, LLC, part of Springer Nature and Neurocritical Care Society

Neurocritical care is a growing clinical discipline which has matured into a full-fledged and recognized multiprofessional subspecialty of critical care medicine. We can say without a doubt that neurocritical care has gone through puberty and adolescence, and now has reached adulthood. The Neurocritical Care Society (NCS) is made up of more than 2000 members from more than 47 countries and has been promoting neurocritical care and supporting its members for over a decade [1]. The vision of NCS is to provide and advocate for the highest quality of care for patients with critical neurological illness throughout the world. NCS has been instrumental at improving professional collaboration between physicians, nurses, pharmacists, and other healthcare practitioners. In addition, NCS has improved neurocritical care education by drafting multi-disciplinary guidelines of various diseases such as subarachnoid hemorrhage $(\mathrm{SAH})$, status epilepticus, and severe acute ischemic strokes (AIS). Physician neurointensivists and neurocritical care fellowship training programs can now obtain certification and accreditation in the USA via the United Council of Neurological Subspecialties, and many national organizations, such as Leapfrog, have recognized the specialty. More recently, the American Board of Psychiatry and Neurology (ABPN) has approved board certification for physician neurointensivists [2]. This latest move by ABPN will bring neurocritical care training to the same level as any other critical care training and certification in the USA. Exciting and very welcoming news indeed.

*Correspondence: jsuarez5@jhmi.edu

Division of Neurosciences Critical Care, Departments of Anesthesiology and Critical Care, Neurology, and Neurosurgery, The Johns Hopkins University School of Medicine, 1800 Sheikh Zayed Building, 3014C, Baltimore, MD 21287, USA

This comment refers to the article available at https://doi.org/10.1007/ s12028-018-0601-1.
There also is important evidence suggesting that the availability of organized neurocritical care teams made up of fellowship-trained neurointensivists, neurocritical care nurses and pharmacists, and other dedicated healthcare professionals is associated with improved outcomes, reduced length of stay and resource utilization, and decreased costs for patients with intracerebral hemorrhage, AIS, SAH, and trauma [3-6]. However, these specialized teams are concentrated in large academic institutions which usually have invested significant resources to build dedicated neurocritical care units (NCCUs). In fact, it has been shown that only one-third of the US population has access to neurocritical care by ground within $90 \mathrm{~min}$ and that there are significant regional variations with some areas having much better access than others [7]. These data raise several important concerns which remain to be answered, including the type of patients who need immediate prehospital transportation to facilities with NCCUs, the determination of the appropriate number of neurocritical care beds for a specific population, and what would be the ideal time frame to get a patient to such specialized units to optimize outcomes, to name but a few. One way to begin to address these vital questions is to establish and agree on standards for NCCUs. Whereas there are pathways for certification of comprehensive stroke and trauma centers which incorporate neurocritical care, there is no clear description of what such care should consist of or where it should be delivered [8, 9]. In 2015, NCS established a multi-disciplinary writing group to identify resources and standards by which to designate adult NCCUs. This writing group has put together a statement that has been endorsed and approved by the NCS Board of Directors and has been published in this issue of Neurocritical Care [10].

The most important recommendation from the NCSappointed writing group is the designation of level-based

\section{Springer}


classification of NCCUs. Specifically, the document calls for 3 types of NCCUs: level I, II, and III. A level I NCCU would be a receiving center for patients with the most complex neurological catastrophes who require the most comprehensive neurocritical care. A level II NCCU would be equipped to stabilize acutely ill patients and safely manage stable neurocritical care processes, while maintaining a close relationship and transfer agreements with level I units. A level III NCCU could provide emergent evaluation and stabilization of patients with neurological emergencies and transfer them to level II or III units after the initial management. The authors have created a list of specific requirements for each NCCU level designation, which should serve as the guiding starting point for regulatory and governmental agencies.

All neurocritical care practitioners should applaud and support this NCS initiative. It was high time that the neurocritical care community began addressing NCCU organization. We envision that this level-based classification of NCCUs will help improve current systems of care and patient outcome. However, several uncertainties remain: Who should be in charge of coordinating this level-based classification? What metrics and methodology should be used to benchmark the quality of care delivered in those NCCUs? How are we going to ensure that gaps in disparity of access to NCCUs begin to close rather than widen? All these are great research and quality improvement initiatives for the new generation of neurocritical care practitioners to address. This is a call to arms; the future of neurocritical care depends on it.

Published online: 8 October 2018

\section{References}

1. https://www.neurocriticalcare.org/home. Accessed 14 Sept 2018

2. https://www.abpn.com/new/abpn-announces-new-neurocritical-carecertificate/. Accessed 14 Sept 2018.

3. Suarez II, Zaidat OO, Suri MF, et al. Length of stay and mortality in neurocritically ill patients: impact of a specialized neurocritical care team. Crit Care Med. 2004;32:2311-7.

4. Bershad EM, Feen ES, Hernandez OH, Suri MF, Suarez JI. Impact of a specialized neurointensive care team on outcomes of critically ill acute ischemic stroke patients. Neurocrit Care. 2008;9:287-92.

5. Diringer MN, Edwards DF. Admission to a neurologic/neurosurgical intensive care unit is associated with reduced mortality rate after intracerebral hemorrhage. Crit Care Med. 2001;29:635-40.

6. Varelas PN, Schultz L, Conti M, Spanaki M, Genarrelli T, Hacein-Bey L. The impact of a neuro-intensivist on patients with stroke admitted to a neurosciences intensive care unit. Neurocrit Care. 2008;9:293-9.

7. Ward MJ, Sutter LA, Branas CC, Adeoye O, Albright KC, Carr BG. Geographic access to US neurocritical care units registered with the neurocritical care society. Neurocrit Care. 2012;16:232-40.

8. https://www.jointcommission.org/certification/advanced_certificat ion_comprehensive_stroke_centers.aspx. Accessed 14 Sept 2018.

9. https://www.facs.org/quality-programs/trauma/about-trauma. Accessed 14 Sept 2018.

10. Moheet AM, Livesay SL, AbdelhakT, BleckTP, Human T, Karanja N, et al. Standards for neurologic critical care units: a Neurocritical Care Society statement. Neurocrit Care. 2018. https://doi.org/10.1007/s12028-0180601-1. 